\title{
Vibrational studies of the disulfide group in proteins Part IV. SS and CS stretch frequencies of known peptide and protein disulfide bridges
}

\author{
Weili Qian, Wenyun Zhao ${ }^{1}$ and Samuel Krimm \\ Biophysics Research Division and Department of Physics, University of Michigan, Ann Arbor, \\ $M I 48109$ (USA)
}

(Received 11 April 1991)

\begin{abstract}
We have used our previously derived ab initio disulfide and empirical polypeptide force fields to calculate SS and CS stretch frequencies of disulfide bridges in a cyclic octapeptide and six proteins of known structure. Comparisons with Raman spectra show that the observed bands are very well reproduced by the normal mode calculations, thus validating the use of these force fields in studying detailed correlations between such spectra and disulfide bridge geometry.
\end{abstract}

\section{INTRODUCTION}

The SS stretch, $\nu$ (SS), and CS stretch, $\nu(\mathrm{CS})$, frequencies in the Raman spectrum have been empirically correlated with the conformations of S-S bridges in proteins for many years [1-6]. While useful at a preliminary level, a more quantitative analysis of this relationship is needed if reliable spectrastructure correlations are to be made, and this requires normal mode calculations of peptide structures containing the disulfide bridge. Previous calculations to model such structures were done primarily on linear [7] or cyclic [8] dialkyl disulfides, although in one calculation [9] the bridge wàs simulated by attaching $\mathrm{C}$ and $\mathrm{N}$ atoms to the $\mathrm{C}^{\alpha}$ atoms. However, our ab initio disulfide force field studies $[10 ; 11]$ have shown $[12]$ that $\nu(\mathrm{SS})$ and $\nu(\mathrm{CS})$ depend on the exact $\mathrm{C}^{\alpha}$ atom structures as well as the dihedral angles $\tau$ (HCCS) $\left(\chi^{1}\right.$, taken as $\tau\left(\mathrm{NC}^{\alpha} \mathrm{C}^{\beta} \mathrm{S}\right)$ in the peptide case), $\tau(\mathrm{CCSS})\left(\chi^{2}\right)$, and $\tau(\mathrm{CSSC})\left(\chi^{3}\right)$, and thus undoubtedly on the $\phi$ and $\psi$ dihedral angles of the peptide groups around the disulfide bridge. Therefore, it is necessary to examine a more realistic

${ }^{1}$ Present address: Department of Chemistry, University of South Carolina, Columbia, SC 29208, USA. 
structure in which the effect of the geometry of the polypeptide chain is taken into account. In addition, the scaled ab initio disulfide force field [11,12] should be used in such calculations since it provides more reliable results than previous empirical force fields [7-9].

Prior to such a more general study, we thought it would be useful to test our scaled ab initio force field on peptide and protein disulfide bridges whose structures are known from single crystal X-ray studies and for which comparable Raman data are available. We have chosen to examine the S-S bridges of the cyclic octapeptide cyclo (Cys-Gly-Pro-Phe $)_{2}$ [13] (c(CGPF) $)_{2}$ ), for which we have obtained Raman spectra, and several proteins for which $X$-ray structures [14] and Raman data are available: actinoxanthin [9], bovine pancreatic trypsin inhibitor [4] (BPTI), lysozyme [15], ribonuclease A [16], insulin [17] and phospholipase $A_{2}$ [9]. As will be seen, the predicted frequencies agree very well with the observed bands, thus providing a secure base for investigating the more general dependence of spectra on conformation [18].

\section{DISULFIDE BRIDGE MODEL}

In modeling the peptide disulfide bridge in a normal mode calculation, the first question to be answered is how large a structure is needed to reproduce the observed $\nu$ (SS) and $\nu$ (CS). The earlier approximate model with 16 atoms [9] cannot reproduce the $\nu(\mathrm{CS})$ when just a heavy atom (C or $\mathrm{N})$ is placed trans to an $\mathrm{S}$ atom across the $\mathrm{CC}$ bond [12]. This difficulty is resolved by a model with 28 atoms, in which a $\mathrm{CH}_{3}$ group is used instead of a single $\mathrm{C}$ atom [12]. However, as we used more realistic models to simulate the disulfide bridge in proteins, namely more atoms in the calculation, we found that $\nu(\mathrm{SS})$ and especially $\nu$ (CS) changed by dozens of wavenumbers. Therefore, we increased the number of atoms until $\nu$ (SS) and $\nu$ (CS) did not change by more than a couple of wavenumbers with further additions of atoms. In this way, we found that the 32-atom model $\left(\mathrm{SCH}_{2} \mathrm{CH}_{\backslash}-\mathrm{CONHC}_{2}\right.$ was a satisfactory representation of the $\mathrm{S}-\mathrm{S}$ bridge. A total of 102 internal coordinates are involved in this model.

For the disulfide group, the optimized ab initio geometry of diethyl disulfide was used, since the scaled ab initio force field was determined for this structure [11]. Since these calculations were only done for conformers having $\tau(\mathrm{CS})$ near $0^{\circ}$ (cis, C), $60^{\circ}$ (gauche, G), $120^{\circ}$ (skew, S), $180^{\circ}$ (trans, T), $-120^{\circ}$ $\left(\mathrm{S}^{\prime}\right)$ and $-60^{\circ}\left(\mathrm{G}^{\prime}\right)$ (the prime indicating a negative angle), and actual values in proteins cover a much broader range, we have defined other conformational states and used average geometries for them. Following earlier practice [5], we designate states for $\tau(\mathrm{CS})$ near $30^{\circ}$ (A) and $90^{\circ}$ (B); we also introduce another state near $150^{\circ}$ (D). (Note that we still follow the convention of labeling the preferred conformation about $\tau(\mathrm{SS})$ as $\mathrm{G}$, even though this dihedral angle 
is near $90^{\circ}$.) The geometries of the A, B and D states were taken as averages of those of their neighboring conformers, the $B$ matrix being calculated from such averaged geometries (averaging the corresponding $\boldsymbol{B}$ matrices did not give reasonable results). In the case of the peptide group, since we used an empirical force field [19], we utilized the standard peptide geometry [19] for which this force field was refined.

The ab initio force constants were obtained on diethyl disulfide with righthanded $(G)$ chirality [11], but disulfide bridges in proteins occur with about equal probability in left-handed $\left(\mathrm{G}^{\prime}\right)$ chiral arrangements [20]. It is therefore necessary to derive a force field for the left-handed disulfide bridge from that obtained for the right-handed bridge [12]. This is straightforward, however, since the potential function in internal coordinates, $V=\sum_{i j} F_{i j} R_{i} R_{j}$, is invariant to such a mirror reflection, which only inverts the signs of the torsion and outof-plane bend coordinates. Since the latter do not occur in the S-S bridge, we only need to change the sign of the $F_{i j}$ in the original force field [12] when only one of the $R$ values is a torsion internal coordinate in order to obtain the corresponding $F_{i j}$ for the left-handed bridge. The peptide moiety is always in the L configuration, and thus our previously refined peptide force field [19] is applicable as it is.

The values of the S-S bridge force constants used in the calculations were obtained by the method previously used [11]. For example, the force constants for $\mathrm{SG}^{\prime} \mathrm{B}^{\prime} \mathrm{c}(\mathrm{CGPF})_{2}$ (representing the $\chi_{1}^{2} \chi^{3} \chi_{2}^{2}$ angles) were taken as average values of those of the $S^{\prime}$ GG and $S^{\prime}$ GS conformers, those of $S^{\prime} G G$, for instance, being appropriate averages of the ab initio values calculated for the $\mathrm{S}^{\prime} \mathrm{GS}^{\prime}$ and GGG conformers [11].

The peptide group force constants were taken from our empirical force field [19] (the values for $\beta$-poly (L-alanine) were used). The diagonal force constants for the region where the S-S bridge and the polypeptide chain join, for example $\mathrm{C}^{\alpha} \mathrm{C}^{\beta}$ and $\mathrm{C}^{\alpha} \mathrm{H}$ stretch, and for the interactions corresponding to the two parts, for example $\mathrm{C}^{\alpha} \mathrm{C}^{\beta}$ stretch $/ \mathrm{NC}^{\alpha} \mathrm{H}$ bend and $\mathrm{C}^{\alpha} \mathrm{C}^{\beta}$ stretch $/ \mathrm{CC}^{\alpha} \mathrm{H}$ bend, were taken as the average of the scaled ab initio force constant for diethyl disulfide and the empirical force constant for the polypeptide chain.

\section{RESULTS AND DISCUSSION}

We compare below the $\nu(\mathrm{SS})$ and $\nu(\mathrm{CS})$ calculated for the $27 \mathrm{~S}-\mathrm{S}$ bridges in the seven molecules studied. Only modes with contributions from SS or CS stretch $\geqslant 15$ are included. In the case of CS stretch, the other components are mainly from peptide-group amide $\mathrm{V}$ contributions ( $\mathrm{NH}$ out-of-plane bend and $\mathrm{CN}$ torsion), which usually give rise to very weak bands in the Raman but probably gain intensity from the CS stretch contribution. At present, independent assignments are not available for the $\nu(\mathrm{CS})$ modes, so our correlations based on frequency proximity should be regarded as suggestive in the light of 
the agreement for alkyl disulfides [11] and the discussions concerning other expected modes.

Cyclo(Cys-Gly-Pro-Phe $)_{2}$

A Raman spectrum of crystalline c(CGPF) ${ }_{2}$ (Spex 1403 spectrometer, $\mathrm{Ar}^{+}$ laser $514.5 \mathrm{~nm}$ excitation) in the region from $450-850 \mathrm{~cm}^{-1}$ is shown in Fig. 1. A comparison of observed and calculated bands is given in Table 1.

This cyclic peptide has one S-S bridge whose conformation is $\mathbf{S G}^{\prime} \mathbf{B}^{\prime}$ [13]. The main calculated $\nu$ (SS) of $506 \mathrm{~cm}^{-1}$ is obviously in excellent agreement with the very strong, sharp and symmetrical band at the same frequency. Another mode with a smaller (but predominant) contribution of $\nu(\mathrm{SS})$ is predicted at $534 \mathrm{~cm}^{-1}$, with the only other calculated mode in this region being $527 \mathrm{C}^{\alpha} \mathrm{CN} \mathrm{d}(25) \mathrm{C}^{\alpha} \mathrm{CO} \mathrm{d}(11)$ (d=deformation). Since Phe exhibits a band at $525-530 \mathrm{~cm}^{-1}$ [1], it is possible that the three weak bands observed at 515,527 and $541 \mathrm{~cm}^{-1}$ should be assigned to $P$ he and the two predicted modes of the $\mathrm{S}-\mathrm{S}$ bridge. On this basis, we associate the $541 \mathrm{~cm}^{-1}$ band with the second $\nu$ (SS) mode.

The $623 \mathrm{~cm}^{-1}$ band is clearly the ring mode of Phe side chain [1]. In the region between this band and $700 \mathrm{~cm}^{-1}$, five bands are predicted for our S-S bridge model: $654 \mathrm{C}^{\alpha} \mathrm{C} \mathrm{s}(17) \mathrm{CS} \mathrm{s}(16) ; 658 \mathrm{C}^{\alpha} \mathrm{C} \mathrm{s}(21) \mathrm{CO}$ ob(13); $672 \mathrm{CS}$ $\mathrm{s}(54) \mathrm{CO} o b(27) ; 679 \mathrm{CO}$ ob(63) CS s(13); and $690 \mathrm{CO} \mathrm{ob(65)} \mathrm{(s=stretch,}$ $\mathrm{ob}=$ out-of-plane bend). The assignments of the 656 and $666 \mathrm{~cm}^{-1}$ bands to the indicated $\nu$ (CS) modes are clearly reasonable. Between 700 and $800 \mathrm{~cm}^{-1}$, bands are predicted at $751 \mathrm{CS} \mathrm{s}(55), 762 \mathrm{CO} \mathrm{ob(37)} \mathrm{and} 772 \mathrm{CO} \mathrm{ob}(21)$. It is difficult to be certain of the assignment of the observed bands at 748, 759, 766 and $786 \mathrm{~cm}^{-1}$ to these modes, but if the $748 \mathrm{~cm}^{-1}$ band is associated with the Phe side chain [1] then the $759 \mathrm{~cm}^{-1}$ band is reasonably assigned as a $\nu(\mathrm{CS})$ and the others as $\mathrm{CO}$ ob modes.

\section{Actinoxanthin}

This protein has two S-S bridges, $B^{\prime} G^{\prime}{ }^{\prime}$ and $G^{\prime} G^{\prime} T$, and its calculated modes are given in Table 2. Only two bands in the $\nu$ (SS) region were reported [9], and it can be seen that the observed bands at 510 and $524 \mathrm{~cm}^{-1}$ are in excellent agreement with the calculated frequencies of the main SS s modes. The early empirical correlation [2] of a GGT conformation with a $\nu$ (SS) near $525 \mathrm{~cm}^{-1}$ is well reproduced here, as it was for the dialkyl disulfides [11]. Another (weaker) $\nu$ (SS) mode is predicted near $530 \mathrm{~cm}^{-1}$, but current data [9] are not available to determine if it is observed. 


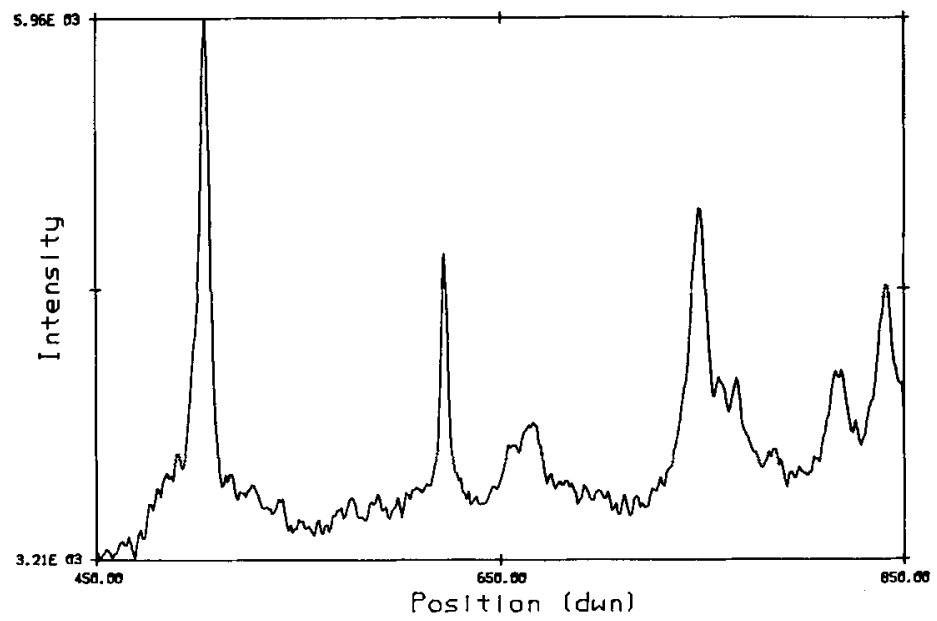

Fig. 1. Raman spectrum of crystalline cyclo (Cys-Gly-Pro-Phe $)_{2}$ from $450-850 \mathrm{~cm}^{-1}$.

\section{TABLE 1}

Disulfide bridge frequencies (in $\mathrm{cm}^{-1}$ ) of cyclo (Cys-Gly-Pro-Phe) ${ }_{2}$

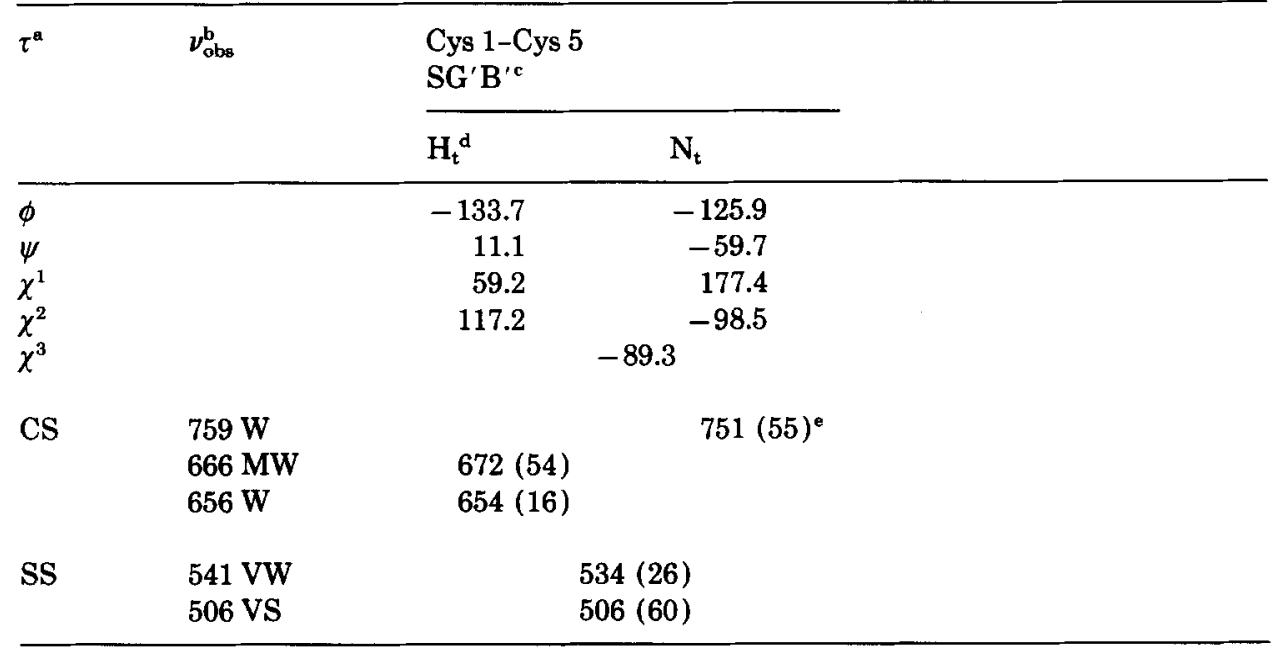

aihedral angles: $\phi=\tau\left(\mathrm{NC}^{\alpha}\right) ; \psi=\tau\left(\mathrm{C}^{\alpha} \mathrm{C}\right) ; \chi^{1}=\tau\left(\mathrm{C}^{\alpha} \mathrm{C}^{\beta}\right) ; \chi^{2}=\tau\left(\mathrm{C}^{\beta} \mathrm{S}\right) ; \chi^{3}=\tau(\mathrm{SS}) . \mathrm{CS}=\mathrm{CS}$ stretch frequencies, $\mathrm{SS}=\mathrm{SS}$ stretch frequencies.

'Our spectra; $\mathrm{S}=$ strong, $\mathrm{M}=$ medium, $\mathrm{W}=$ weak, $\mathrm{V}=$ very.

${ }^{c} \mathrm{XYZ}=\chi_{1}^{2} \chi^{3} \chi_{2}^{2}$.

dAtom trans to $S$ across $\mathrm{C}^{\alpha} \mathrm{C}^{\beta}$ bond; $\mathrm{H}_{\mathrm{t}}, \chi^{1} \approx 60^{\circ} ; \mathrm{C}_{\mathrm{t}}, \chi^{1} \approx-60^{\circ} ; \mathrm{N}_{\mathrm{t}}, \chi^{1} \approx 180^{\circ}$.

Potential energy contribution from appropriate bond in parentheses: $\geq 15$ for SS and CS stretch. 
TABLE 2

Disulfide bridge frequencies (in $\mathrm{cm}^{-1}$ ) of actinoxanthin

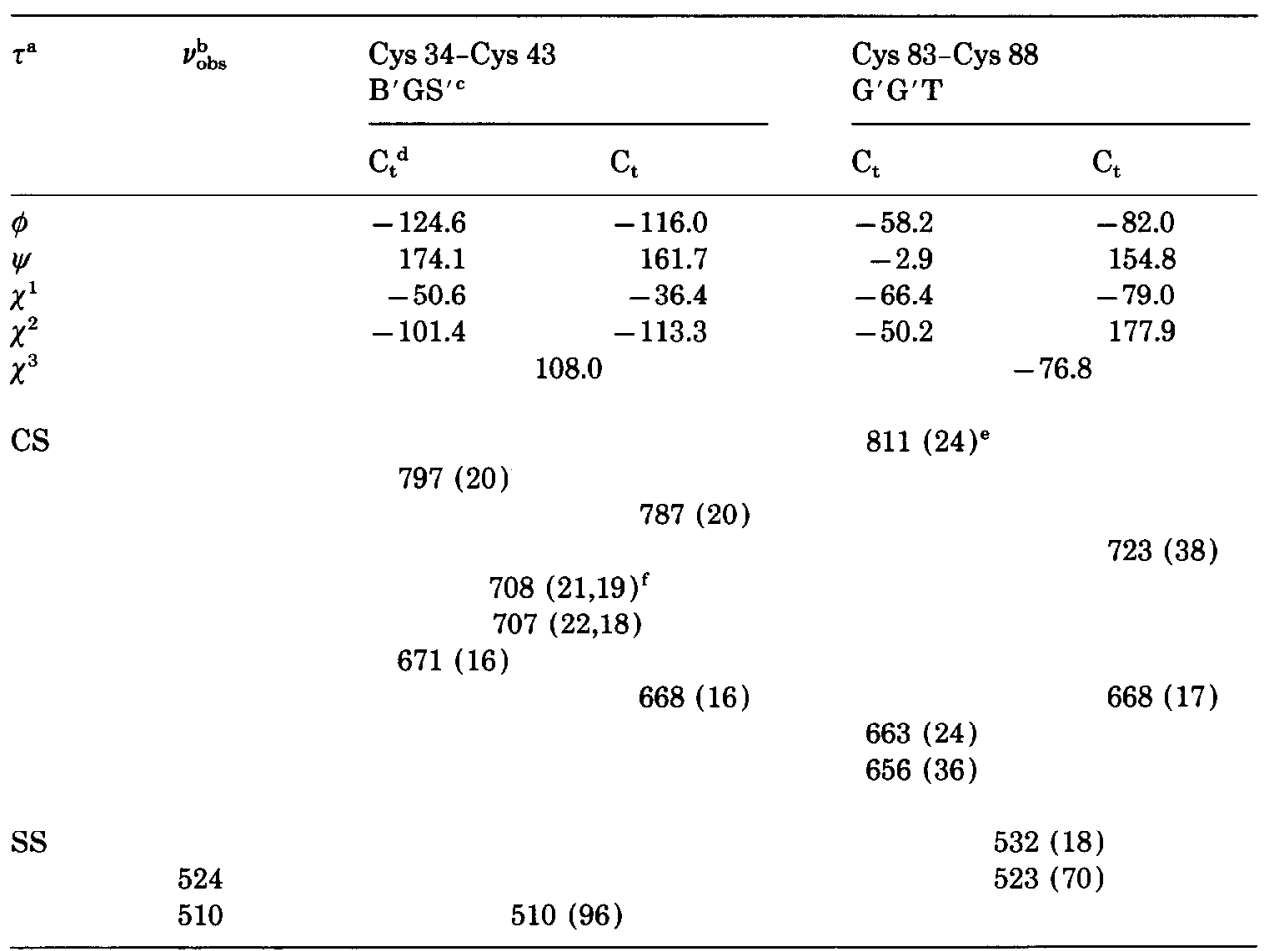

a,c,d,e See Table 1.

${ }^{\text {bRef. } 9 .}$

'First number refers to $\operatorname{CS}\left(\chi_{1}^{2}\right)$, second to $\operatorname{CS}\left(\chi_{2}^{2}\right)$.

Bovine pancreatic trypsin inhibitor

BPTI has three S-S bridges, whose conformations can be designated $B^{\prime} G^{\prime} G^{\prime}$, SGS' and $B^{\prime} G^{\prime} B^{\prime}$. The calculated frequencies are given in Table 3 , and compared with the observed bands in the $\nu(\mathrm{SS})$ and $\nu(\mathrm{CS})$ regions [4]. As before, the predicted $\nu(\mathrm{SS})$ modes are in excellent agreement with the observed band at $508 \mathrm{~cm}^{-1}$.

A complication arises in the assignments of the $\nu$ (CS) modes, since BPTI has one methionine residue that gives rise to such modes in similar regions to those of the $\mathrm{S}-\mathrm{S}$ bridge. Using model compounds, it has been possible to relate the $\nu$ (CS) frequencies to the $\mathrm{C}^{\alpha} \mathrm{C}^{\beta}-\mathrm{C}^{\gamma} \mathrm{S}$ and $\mathrm{C}^{\beta} \mathrm{C}^{\gamma}-\mathrm{SCH}_{3}$ dihedral angles in this side chain [21]: $\mathrm{TG}, 698 \mathrm{~S}$ and $748 \mathrm{~W} \mathrm{~cm}^{-1}$; TT, $718 \mathrm{~S}$ and $762 \mathrm{~W} \mathrm{~cm}^{-1}$; GG, $648 \mathrm{~S}$ and $724 \mathrm{~S} \mathrm{~cm}^{-1}$, and $\mathrm{GT}, 667 \mathrm{~W} \mathrm{~cm}^{-1}$ (relative intensities are for liquid methyl propyl sulfide [21]). The Met residue in BPTI can adopt two confor- 
TABLE 3

Disulfide bridge frequencies (in $\mathrm{cm}^{-1}$ ) of bovine pancreatic trypsin inhibitor

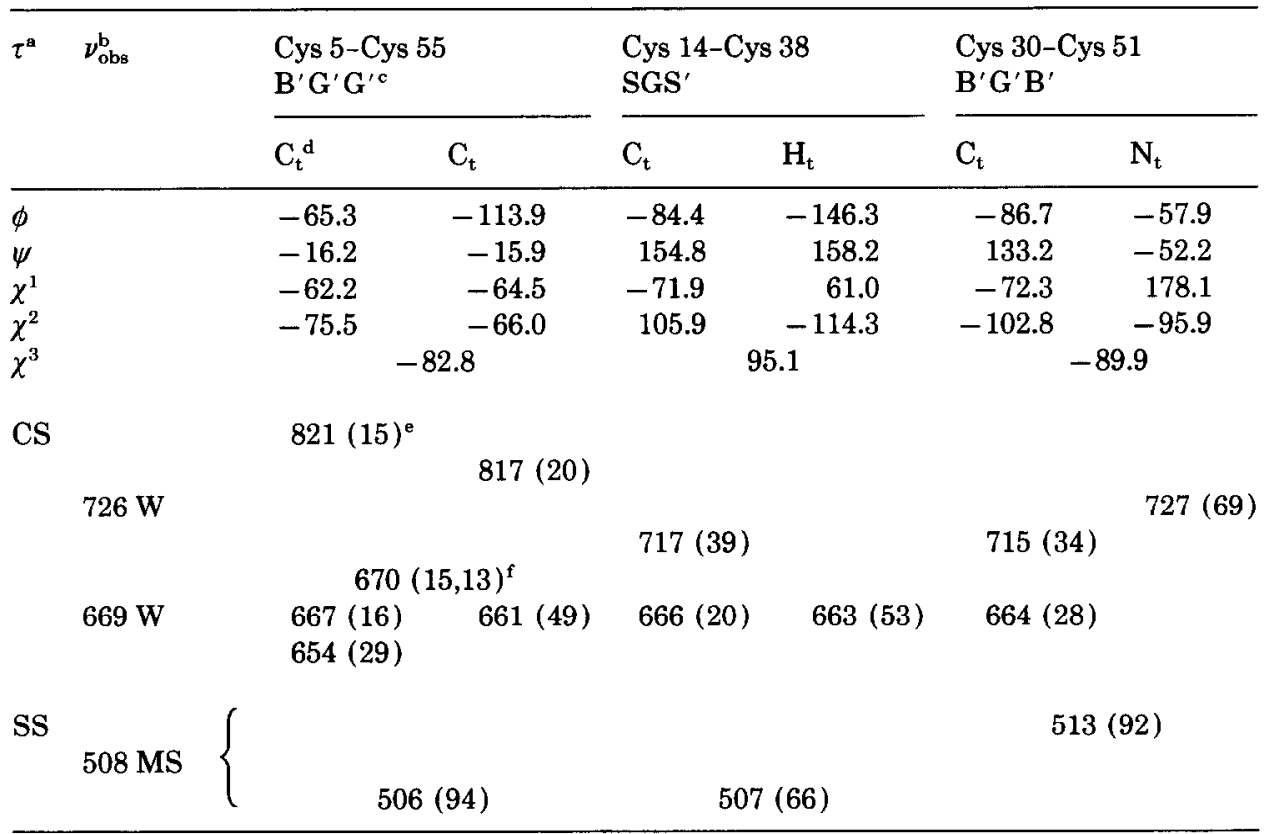

a,c,d,e,f See Table 2.

${ }^{b}$ Ref. 4; $S=$ strong, $M=$ medium, $W=$ weak.

mations [14], with $\tau\left(\mathrm{C}^{\beta} \mathrm{C}^{\gamma}\right)$ and $\tau\left(\mathrm{C}^{\gamma} \mathrm{S}\right)$ of $-64.9^{\circ}$ and $-69.8^{\circ}$, or of $-170.0^{\circ}$ and $-49.6^{\circ}$, corresponding to conformations of GG and TG. We thus expect their $\nu(\mathrm{CS})$ contributions to be near 648 and $724 \mathrm{~cm}^{-1}$ and near 698 and 748 $\mathrm{cm}^{-1}$, respectively. While it is possible that the observed $726 \mathrm{~W} \mathrm{~cm}^{-1}$ band of BPTI could be contributed to by $\nu(\mathrm{CS})$ of both Met and the S-S bridge (the presence of $a \approx 650 \mathrm{~cm}^{-1}$ band would give support to the former assignment), it is clear that the $669 \mathrm{~cm}^{-1}$ band should be associated only with the S-S bridge. Its frequency is quite consistent with predicted $\nu(\mathrm{CS})$ modes. We note that this value is considerably lower than the $700-745 \mathrm{~cm}^{-1}$ range previously suggested [2] for such $\mathrm{C}_{\mathrm{t}}$ conformations ( $\mathrm{C}$ trans to $\mathrm{S}$ across the $\mathrm{C}^{\alpha} \mathrm{C}^{\beta}$ bond).

\section{Lysozyme}

Lysozyme has four S-S bridges, designated $\mathrm{G}^{\prime} \mathrm{G}^{\prime} \mathrm{A}^{\prime}, \mathrm{B}^{\prime} \mathrm{G}^{\prime} \mathrm{G}^{\prime}, \mathrm{BGG}^{\prime}$ and TGG; their calculated frequencies are given in Table 4 and compared with observed bands [15]. There are two Met residues in lysozyme [14], whose conformations are $-76.3^{\circ}$ and $-98.8^{\circ}(\mathrm{GG})$ and $-68.8^{\circ}$ and $-64.4^{\circ}(\mathrm{GG})$, which should give rise to $\nu(\mathrm{CS})$ bands near 648 and $724 \mathrm{~cm}^{-1}$ [21]. 


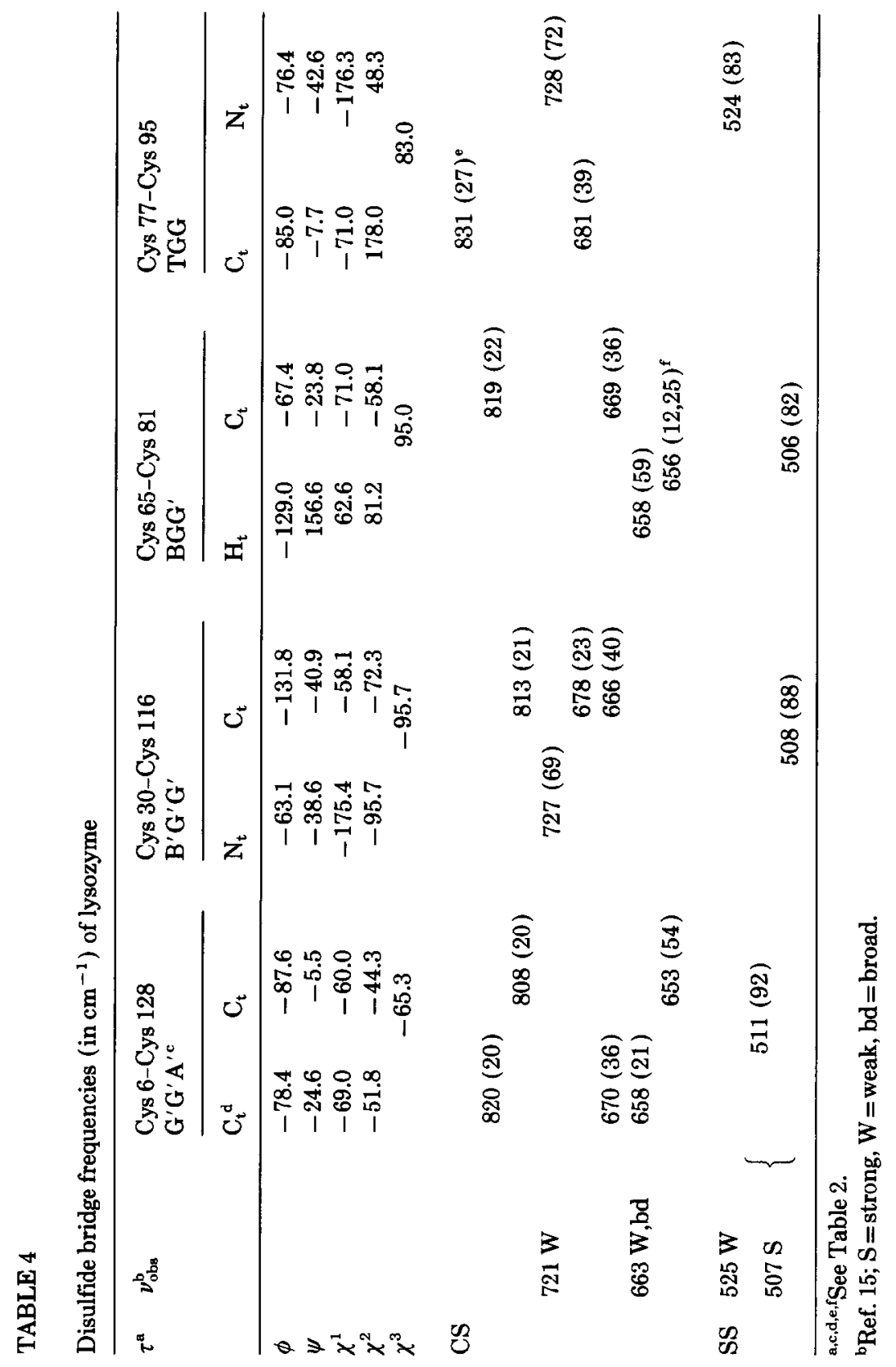


The Raman spectrum of lysozyme exhibits one strong band in the $\nu$ (SS) region, at $507 \mathrm{~cm}^{-1}$, that has been assigned to this mode [15]. Our calculations reproduce this band quite well, but also suggest that there should be a $\nu$ (SS) band near $524 \mathrm{~cm}^{-1}$. Such a band is observed but was not so assigned [15]; we suggest this assignment, consistent with our similar assignment to such a conformation in actinoxanthin (see Table 2).

In the $\nu(\mathrm{CS})$ region, the observed band at $663 \mathrm{~cm}^{-1}$ is unusually broad, consistent with the relatively large range of expected modes for the $\mathrm{S}-\mathrm{S}$ bridges. The only other predicted modes in this region are $663 \mathrm{CO}$ ob (56) for Cys 6Cys 128; $695 \mathrm{CO}$ ob(62) for Cys 30-Cys 116; $692 \mathrm{CO}$ ob(72) for Cys 65-Cys 81; and $695 \mathrm{CO} \mathrm{ob(62)}$ for Cys 77-Cys 95, the last three of which may be assignable to the observed band at $696 \mathrm{~cm}^{-1}$. Again a band is observed at 721 $\mathrm{cm}^{-1}$ that could be associated with $\nu(\mathrm{CS})$ modes of two of the S-S bridges. However, it may also derive from the Met residues; the presence of the 644 $\mathrm{cm}^{-1}$ Tyr band makes it difficult to determine if the $648 \mathrm{~cm}^{-1}$ counterpart is seen.

\section{Ribonuclease A}

Ribonuclease A has four S-S bridges, $B^{\prime} G^{\prime} G^{\prime}, G^{\prime} G^{\prime} G^{\prime}, G^{\prime} G^{\prime} S^{\prime}$ and $G^{\prime} G B$, whose calculated frequencies are compared with observed bands [16] in Table 5 . There are four Met residues in this protein [14], whose conformations are $-49.8^{\circ}$ and $-60.2^{\circ}(\mathrm{GG}) ;-72.1^{\circ}$ and $-59.1^{\circ}(\mathrm{GG}) ;-59.5^{\circ}$ and $-68.0^{\circ}$ (GG); and $-171.4^{\circ}$ and $176.3^{\circ}$ (TT). These should give rise to bands at 648 , $718-724$, and $762 \mathrm{~cm}^{-1}$ [21].

The single strong band at $514 \mathrm{~cm}^{-1}$ is consistent with our calculated $\nu$ (SS) modes. The calculated $\nu(\mathrm{CS})$ modes in the $650-700 \mathrm{~cm}^{-1}$ region comprise all the predicted vibrations for our bridge model, and it therefore seems reasonable to assign observed bands at 654 and $670 \mathrm{~cm}^{-1}$ to these modes, as is the case for the $724 \mathrm{~cm}^{-1}$ band. The only problem is that Met $\nu$ (CS) modes may also be contributing to the 654 and $724 \mathrm{~cm}^{-1}$ bands. Thus, only the assignment of the $670 \mathrm{~cm}^{-1} \nu(\mathrm{CS})$ mode is reasonably secure at present.

\section{Insulin}

The three S-S bridge pairs of insulin have the following comparable conformations: $\mathrm{G}^{\prime} \mathrm{GT}$ and $\mathrm{G}^{\prime} \mathrm{GD}^{\prime}, \mathrm{GGB}^{\prime}$ and $\mathrm{GGB}^{\prime}$, and $\mathrm{G}^{\prime} \mathrm{G}^{\prime} \mathrm{G}^{\prime}$ and $\mathrm{A}^{\prime} \mathrm{G}^{\prime} \mathrm{G}^{\prime}$. Their calculated frequencies are given in Table 6 and compared with observed bands [17]. Insulin has no Met residues, so the $\nu(\mathrm{CS})$ region is not complicated by its modes.

The strong $514 \mathrm{~cm}^{-1}$ band is consistent with calculated $\nu$ (SS) modes of four of the S-S bridges. The other two are expected to have significantly higher frequencies, and we suggest that the very weak band at $530 \mathrm{~cm}^{-1}$ should be 


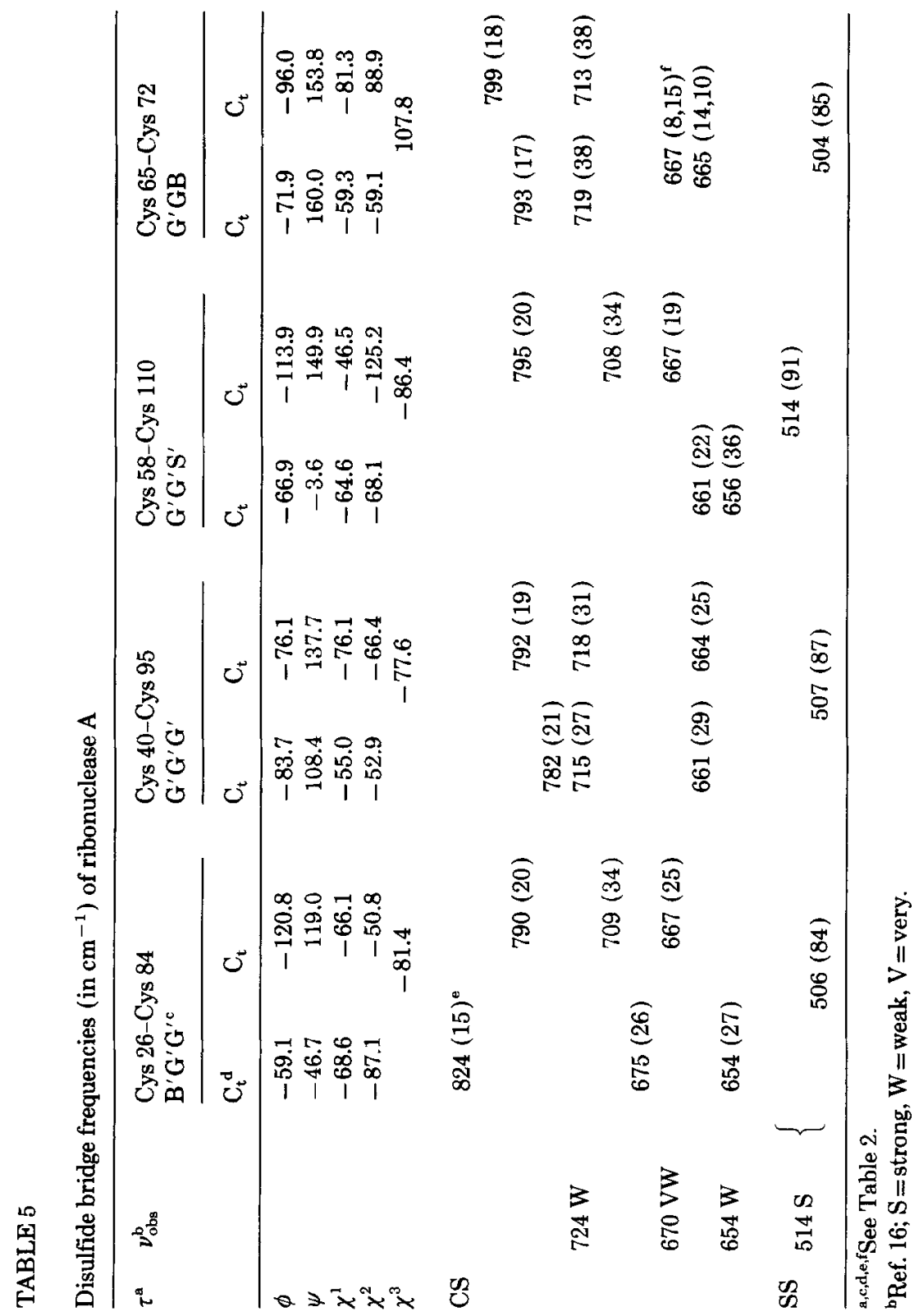




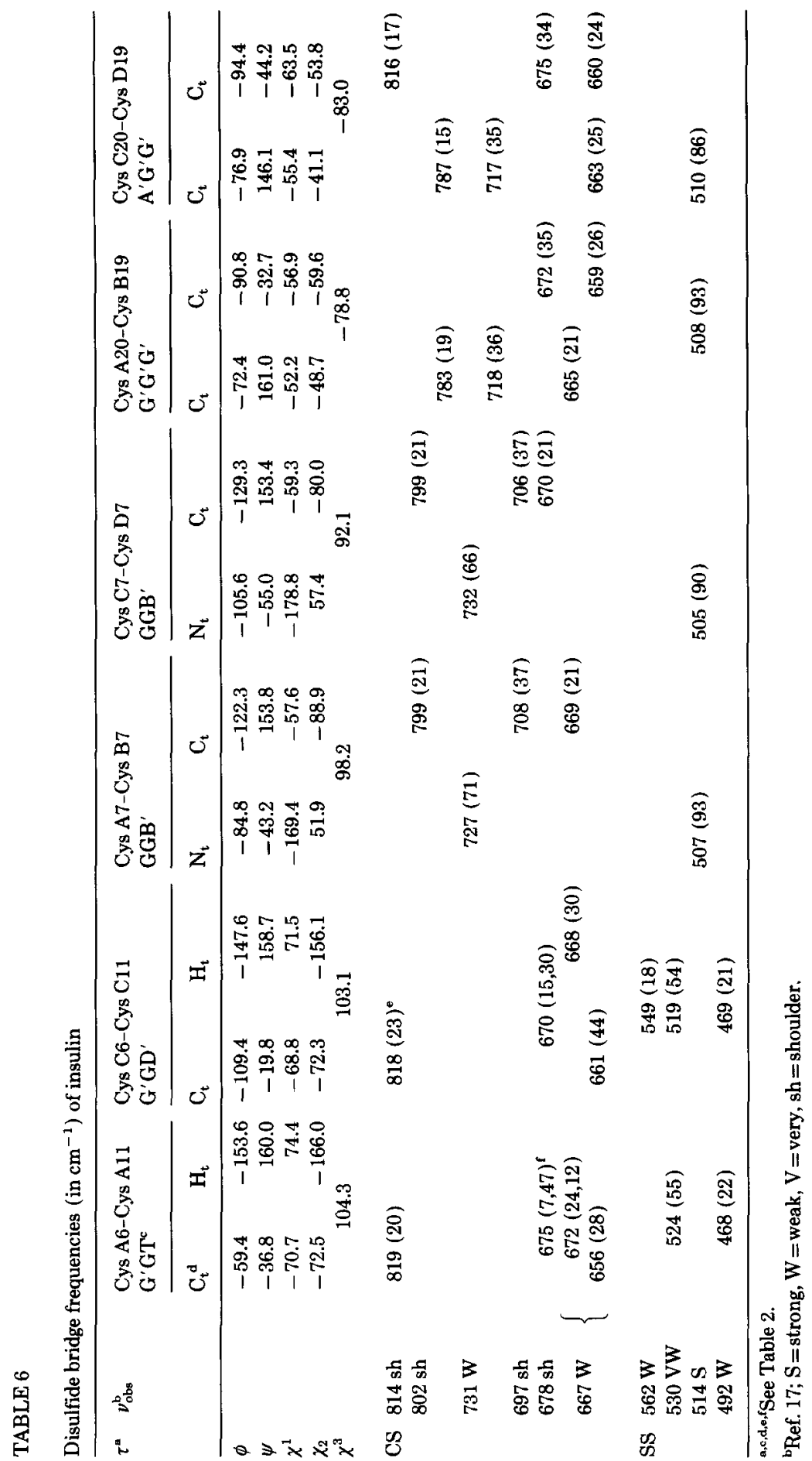




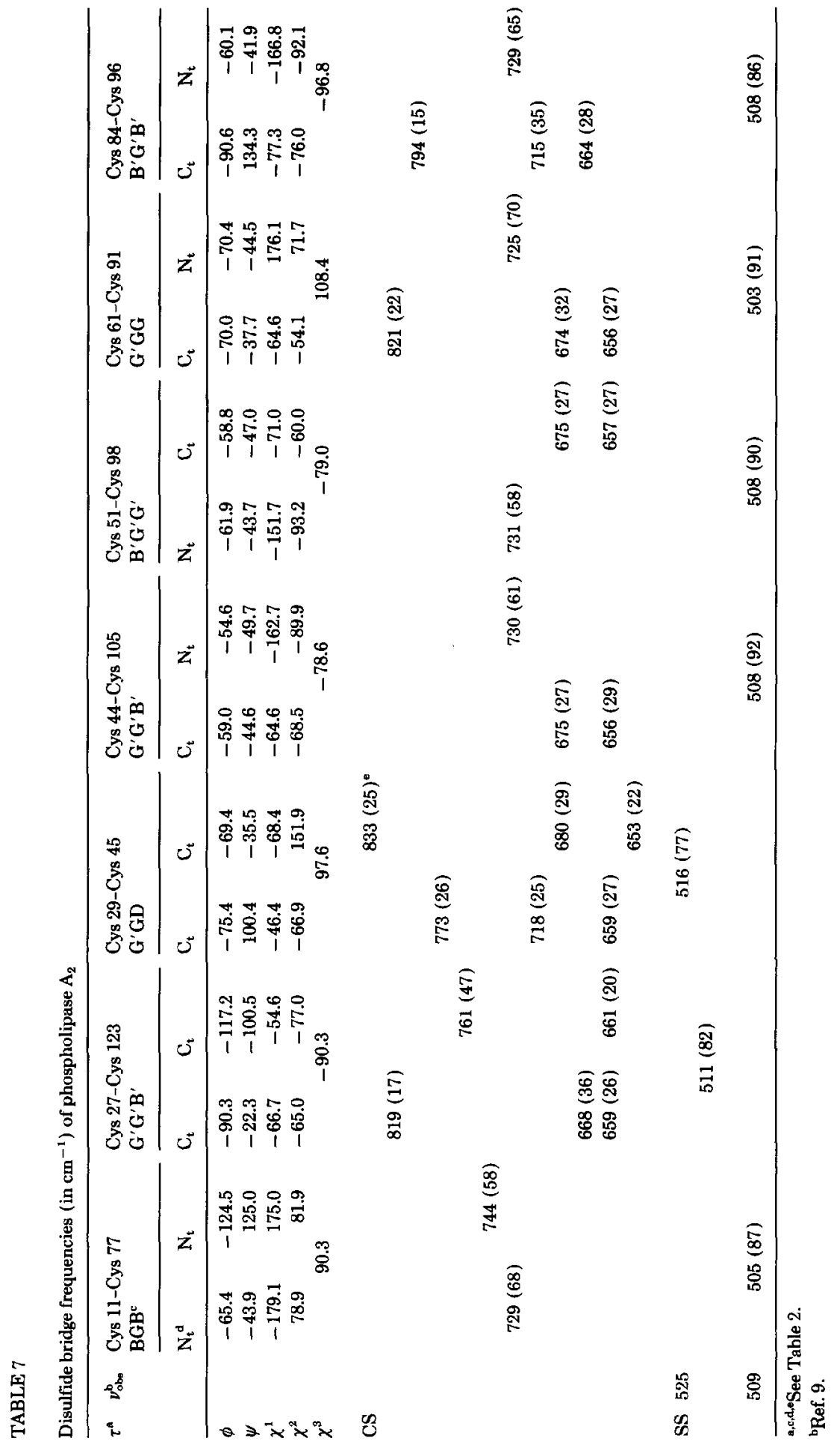


assigned to them. Two other (weak though predominantly $\nu$ (SS)) modes are predicted for these bridges, near 470 and $550 \mathrm{~cm}^{-1}$; it is tempting to suggest (though with caution) that the observed bands at 492 and $562 \mathrm{~cm}^{-1}$ may be assignable to these modes.

The region of calculated $\nu(\mathrm{CS})$ modes is rich with observed bands that are readily assigned to these vibrations. This is particularly true of the 667 and $678 \mathrm{~cm}^{-1}$ bands since no other modes are predicted in this region. Bands in the $\approx 700-800 \mathrm{~cm}^{-1}$ region can also be assigned to predicted $\nu(\mathrm{CS})$ modes, although perhaps with less certainty. It is interesting that the 667 and 731 $\mathrm{cm}^{-1}$ bands have comparable, relatively high intensities; this may be due to the large number of modes contributing to the former and the relatively pure $\nu$ (CS) nature of the latter. This strong $\nu$ (CS) character of bands near 730 $\mathrm{cm}^{-1}$ is characteristic of the $\mathrm{N}_{\mathrm{t}}$ conformation ( $\mathrm{N}$ trans to $\mathrm{S}$ across the $\mathrm{C}^{\alpha} \mathrm{C}^{\beta}$ bond).

\section{Phospholipase $A_{2}$}

Phospholipase $\mathrm{A}_{2}$ has seven $\mathrm{S}-\mathrm{S}$ bridges, whose conformations are BGB, $G^{\prime} G^{\prime} B^{\prime}, G^{\prime} G D, G^{\prime} G^{\prime} B^{\prime}, B^{\prime} G^{\prime} G^{\prime}, G^{\prime} G G$ and $B^{\prime} G^{\prime} B^{\prime}$. Their calculated frequencies are given in Table 7 , and compared with the only reported $\nu$ (SS) modes [9]. The $509 \mathrm{~cm}^{-1}$ band is in excellent agreement with the mean value of $\nu$ (SS) modes predicted for six of the bridges; the $525 \mathrm{~cm}^{-1}$ band is consistent with an assignment to the $\mathrm{G}^{\prime} \mathrm{GD}$ bridge, whose $\nu$ (SS) mode is calculated at $516 \mathrm{~cm}^{-1}$. As in the case of actinoxanthin [9], more detailed studies of the spectra seem desirable.

\section{CONCLUSIONS}

The above results show that calculated $\nu(\mathrm{SS})$ and $\nu(\mathrm{CS})$ frequencies of S$\mathrm{S}$ bridges in seven known peptide and protein structures are in good agreement with observed Raman bands due to these modes. While the $\nu$ (SS) modes are easier to assign, knowledge of the contributions due to specific Met conformations in these molecules permits reasonable inferences regarding the assignments of $\nu$ (CS) modes of the $\mathrm{S}-\mathrm{S}$ bridges.

The agreement between observed and calculated $\nu(\mathrm{CS})$ frequencies in such a wide range of disulfide bridges enables a more secure correlation to be made of these modes with $\chi^{1}$. For $\mathrm{N}_{\mathrm{t}}$ conformations, bands are predicted and observed in the range of $725-755 \mathrm{~cm}^{-1}$. For $\mathrm{C}_{\mathrm{t}}$ conformations, calculated bands have definitely observed counterparts in the regions $650-680$ and $700-725 \mathrm{~cm}^{-1}$, with the probability that predicted bands in the $785-830 \mathrm{~cm}^{-1}$ region can also be observed (cf. insulin, Table 6). For $\mathrm{H}_{\mathrm{t}}$ conformations, the range for observed and calculated frequencies is $650-680 \mathrm{~cm}^{-1}$, which is seen to overlap in part the $\mathrm{C}_{\mathrm{t}}$ range. 
We believe that this study gives support to the validity of our ab initio disulfide [12] and empirical polypeptide [19] force fields in calculating reliable normal modes of disulfide bridges in proteins, and provides a sound basis for developing more detailed correlations between conformation and spectra for such structures [18].

\section{ACKNOWLEDGMENTS}

This research was supported by the Monsanto Company, St Louis, MO, and by NSF grants DMB-8816756 and DMR-8806975. We are indebted to K.D. Kopple for providing a sample of cyclo (Cys-Gly-Pro-Phe $)_{2}$ and wish to thank J. Bandekar for obtaining its Raman spectrum.

\section{REFERENCES}

1 R.C. Lord and N.-T. Yu, J. Mol. Biol., 50 (1970) 509.

2 H. Sugeta, A. Go and T. Miyazawa, Chem. Lett., (1972) 83.

3 H. Sugeta, A. Go and T. Miyazawa, Bull. Chem. Soc. Jpn., 46 (1973) 3407.

4 H. Brunner and M. Holz, Biochim. Biophys. Acta, 379 (1975) 408.

5 H.E. Van Wart and H.A. Scheraga, J. Phys. Chem., 80 (1976) 1812, 1823.

6 T. Takamatsu, I. Harada and K. Hayashi, Biochim. Biophys. Acta, 622 (1980) 189.

7 H. Sugeta, Spectrochim. Acta Part A, 31 (1975) 1729.

8 B.E. Weiss-Lopez, M.H. Goodrow, W.K. Musker and C.P. Nash, J. Am. Chem. Soc., 108 (1986) 1271.

9 A.H. Kuptsov and V.I. Trofimov, J. Biomol. Struct. Dyn., 3 (1985) 185.

10 W. Zhao, J. Bandekar and S. Krimm, J. Am. Chem. Soc., 110 (1988) 6891.

11 W. Zhao and S. Krimm, J. Mol. Struct., 224 (1990) 7.

12 W. Zhav, J. Bandekar and S. Krimm, J. Mol. Struct., 238 (1990) 43.

13 K.D. Kopple, Y.S. Wang, A.G. Cheng and K.K. Bhandary, J. Am. Chem. Soc., 110 (1988) 4168.

14 F.C. Bernstein, T.F. Koetzle, G.J.B. Williams, E.F. Meyer, M.D. Brice, J.R. Rodgers, O. Kennard, T. Shimanouchi and M. Tasumi, The Protein Data Bank: a computer-based archival file for macromolecular structures, J. Mol. Biol., 112 (1977) 535. N.-T. Yu and B.H. Jo, Arch. Biochem. Biophys., 156 (1973) 469.

16 N.-T. Yu and B.H. Jo, J. Am. Chem. Soc., 95 (1973) 5033.

17 N.-T. Yu, B.H. Jo, R.C.C. Chang and J.D. Huber, Arch. Biochem. Biophys., 160 (1974) 614.

18 W. Qian and S. Krimm, to be published.

19 S. Krimm and J. Bandekar, Adv. Protein Chem., 38 (1986) 181.

20 N. Srinivasan, R. Sowdhamini, C. Ramakrishnan and P. Balaram, Int. J. Peptide Protein Res., 36 (1990) 147.

21 N. Nogami, H. Sugeta and T. Miyazawa, Chem. Lett., (1975) 147. 